\title{
EVALUATION OF VOICE ACOUSTIC PARAMETERS RELATED TO THE VOCAL-LOADING TEST IN PROFESSIONALLY ACTIVE TEACHERS WITH DYSPHONIA
}

\section{EWA NIEBUDEK-BOGUSZ, PIOTR KOTYŁO, and MARIOLA ŚLIWIŃSKA-KOWALSKA}

Clinic of Audiology and Phoniatrics

Nofer Institute of Occupational Medicine

Łódź, Poland

\begin{abstract}
Introduction: Teachers are at risk of developing voice disorders. A clinical battery of vocal function tests should include non-invasive and accurate measurements. The quantitative methods (e.g., voice acoustic analysis) make it possible to objectively evaluate voice efficiency and outcomes of dysphonia treatment. Materials and Methods: To identify possible signs of vocal fatigue, acoustic waveform perturbations during sustained phonation were measured before and after the vocal-loading test in 51 professionally active female teachers with functional voice disorders, using IRIS software. All the participants were also subjected to laryngological/phoniatric examination involving videostroboscopy combined with selfestimation by voice handicap index (VHI)-based scale. Results: The phoniatric examination revealed glottal insufficiency with bowed vocal folds in $35.2 \%$, soft vocal nodules in $31.4 \%$, and hyperfunctional dysphonia with a tendency towards vestibular phonation in $19.6 \%$ of the patients. In the VHI scale, $66 \%$ of the female teachers estimated their own voice problems as moderate disability. An acoustic analysis performed after the vocal-loading test showed an increased rate of abnormal frequency perturbation parameters (pitch perturbation quotient (Jitter), relative average perturbation (RAP), pitch period perturbation quotient (PPQ)) compared to the pre-test outcomes. The same was true of pitch-intensity contour of vowel /a:/, an indication of voice instability during sustained phonation. Discussion and Conclusions: The recorded impairments of voice acoustic parameters related to vocal loading provide further evidence of dysphonia. The voice acoustic analysis performed before and after the vocal-loading test can significantly contribute to objective voice examinations useful in diagnosis of dysphonia among teachers..
\end{abstract}

Key words:

Voice acoustic analysis, Vocal loading, Dysphonia, Teachers

\section{INTRODUCTION}

In Poland, the total number of teachers is estimated at nearly 740000 people. This is a specific occupational group encumbered with numerous economic, social, and health problems. One of the major health problems is a predisposition to voice disorders [1,2], which can affect the quality of life and the career of teachers as well as reduce their incomes. Our earlier study performed on 425 female teachers and focused on the prevalence of voice disorders, demonstrated that the prevalence of self-reported symptoms and clinical signs of dysphonia was around 2-3 times higher in Polish female teachers than in non-teachers [3]. The main risk factors for developing occupational dysphonia seem to be lifetime vocal effort, poor technique of phonation and psychological predisposition.

An objective evaluation of vocal function in professional voice users is a major difficulty in diagnosis of dysphonia [4,5]. A clinical battery of tests include laryngovideostro-

Received: This paper was presented at the 3rd Voice Congress, Istanbul, Turkey, 19-22 June 2006.

Received: July 17, 2006. Accepted: December 7, 2006.

Address reprint requests to E. Niebudek-Bogusz, MD, Clinic of Audiology and Phoniatrics, Nofer Institute of Occupational Medicine, św. Teresy 8, 91-348 Łódź, Poland (e-mail: ebogusz@imp.lodz.pl). 
boscopy (LVSS), glottography, aerodynamic test or acoustic analysis [6-8]. Among these methods, voice evaluation via acoustic measurements is particularly promising because it is non-invasive and relatively easy to perform. In the literature, significant differences have been found between acoustic analysis of healthy and dysphonic persons $[9,10]$. Various studies have reported that this method could help distinguish between organic and functional dysphonia [11-13]. Several literature data show usefulness of acoustic tests in predicting the dysphonic severity and evaluating the progress in voice therapy $[9,14,15]$.

The aim of this study was to assess the value of acoustic analysis in diagnosis of functional voice disorders in professionally active female teachers and to compare acoustic parameters before and after the vocal-loading test.

\section{MATERIALS AND METHODS}

A total of 51 professionally active full-time female teachers with functional voice disorders (non-organic dysphonia) were examined. The majority of Polish teachers are women, and the majority of our patients seeking help for voice problems are also women. The mean age of subjects ranged from 28 to 57 years (mean, 41 years). Over a half $(58 \%)$ of the patients were primary school teachers, most of them $(32 \%)$ were teachers of languages. The full-time employment of the teachers ranged from 18 to $30 \mathrm{~h} /$ week (average, $21.4 \mathrm{~h}$ ). The average duration of employment was 16.4 years (ranging from 2 to 32 years, median, 17 years).

All the participants responded to the voice handicap index (VHI) questionnaire, then underwent laryngologic and phoniatric examinations, followed by LVSS and voice acoustic analysis performed before and after the vocalloading test.

\section{Voice Handicap Index (the self-estimation scale of voice disorders)}

The VHI questionnaire contains three subscales: physical, emotional and functional, scored $0-4$, which specify phonation complaints. The low VHI value (0-30 scores) may relate to few (slight) or early voice problems; 30 to
60 scores may represent medium-heavy handicap (i.e., discomfort, vocal fatigue or voice loss) and 61-120 scores may suggest severe voice disorders and an urgent need to perform additional voice examinations.

\section{ENT- phoniatric examination with laryngovideostroboscopy}

Clinical examinations comprised routine laryngological (ENT) examination and perceptual voice evaluation performed by two experienced ENT phoniatricians. The voice assessment also involved determination of maximum phonation time (MPT); each subject was asked to phonate the sustained /a:/ vowel at a comfortable pitch and loudness as long as possible during a single exhalation. The videostroboscopic examination was performed using a rigid scope (Wolf 5052). The results were recorded on a high quality video tape. The vocal fold function was assessed using normal and stroboscopic light during repeated stable phonation of the sustained /i:/ vowel at a comfortable level of pitch and loudness. The following parameters of the phonatory function were examined: the quality of mucosal wave, regularity of vocal fold vibration, amplitude of vocal fold movement and configuration of glottal closure.

\section{Acoustic analysis}

The teachers were asked to produce the sustained /a:/ vowel at comfortable pitch and loudness for at least $5 \mathrm{~s}$. Voice samples were recorded on a computer hard disc using a research quality microphone, and analyzed by IRIS software.

LTAS - long term analysis spectrum of vowel /a:/ in sustained phonation, presented among other things as a graph of $\mathrm{F}_{0}$ (fundamental frequency, $\mathrm{Hz}$ ) vs. loudness (dB) - pitch-intensity contour (P-I contour). The euphonic voice was characterized by normal stability of phonation of the sustained /a:/ vowel, ability to hold comfortable pitch and loudness during sustained phonation, and thus maintained small surface area of P-I contour.

FFT - fast Fourier transform, including a short central segment (0.1-0.6 s) of acoustic waveform of the sustained /a:/ vowel and estimating 17 parameters: average fundamental frequency $\left(\mathrm{F}_{0}\right)$; standard deviation of the fundamental fre- 
quency (sdev $\mathrm{F}_{0}$ ); pitch perturbation quotient (Jitter), relative average perturbation (RAP) and pitch period perturbation quotient (PPQ); amplitude perturbation quotient (Shimmer) and amplitude perturbation quotient (APQ); harmonic perturbation measurements: harmonic perturbation quotient (HPQ); harmonic perturbation quotient for high frequency (HPQh); residual harmonic perturbation quotient (RHPQ); residual harmonic perturbation quotient for high frequency (RHPQh); residual to harmonic $(\mathrm{R} 2 \mathrm{H})$, non-harmonic to harmonic (U2H); U2Hl (for low frequency); $\mathrm{U} 2 \mathrm{Hh}$ (for high frequency), subharmonic to harmonic (S2H); and noise to harmonic ratio (NHR).

\section{Vocal loading test}

The patients were asked to read aloud a text for $30 \mathrm{~min}$ under exposure to $80 \mathrm{dBSPL}$ white noise. The results of loading were assessed with regard to videostroboscopy and acoustic analysis parameters. By comparing the results before and after vocal loading, the result was regarded as positive if at least one parameter evaluated in videostroboscopy was deteriorated, and as negative if none of the parameters was seen altered.

In the acoustic analysis, an outcome was considered positive if, after vocal loading, the patient scored lower on at least one of the following test units:

1. The number of pathological acoustic measurements (of the 17 acoustic parameters measured by IRIS software) increased at least by 2 (Fig. 1).

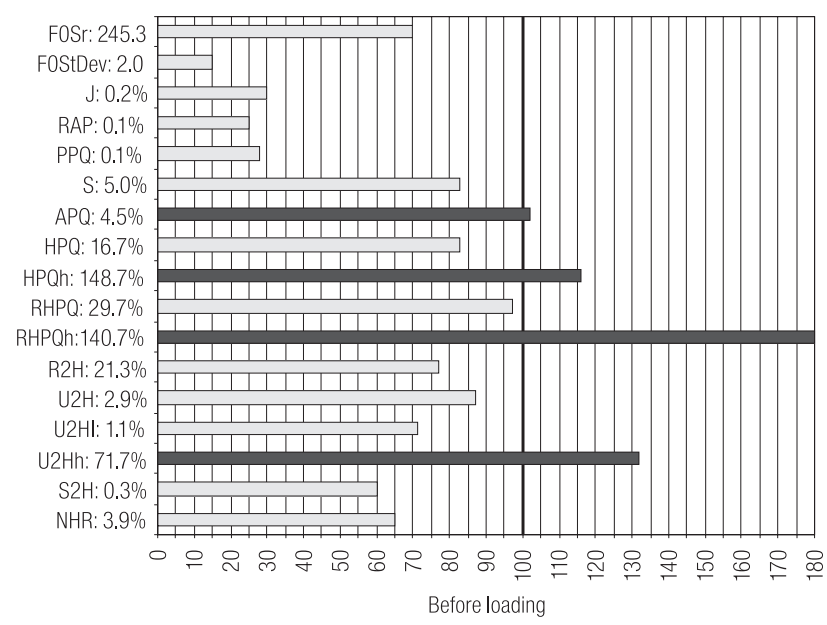

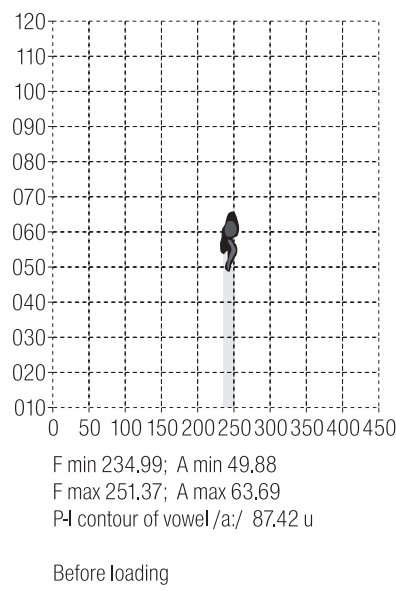

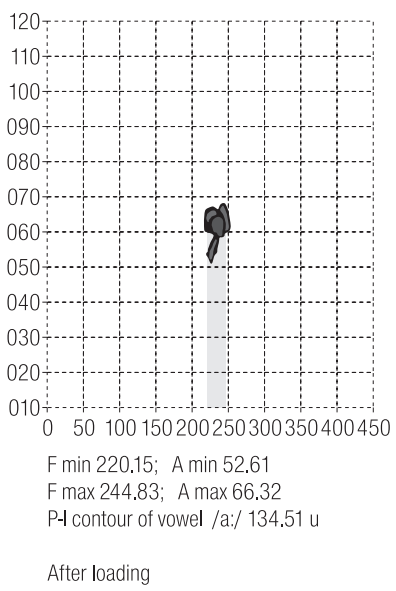

Fig. 2. The positive outcome of vocal loading in acoustic analysis - the Pitch-Intensity contour of vowel /a/: increased from 87.4 to 134.5 units (an indication of voice instability).

2. There was an increase in the P-I contour of vowel /a:/ - an indication of instability of voice (Fig. 2).

\section{Statistical analysis}

The following statistical methods were used to analyze our data:

च the chi-square or Fisher exact test to compare the frequencies or distributions of the categories of discrete variables (e.g., number of abnormal results, number of changes of parameter values or values above and below normal),

a multi-way analysis of variance for dependent variables (repeated measures) for within-group comparison of the effect of vocal loading on the patients' vocal apparatus.

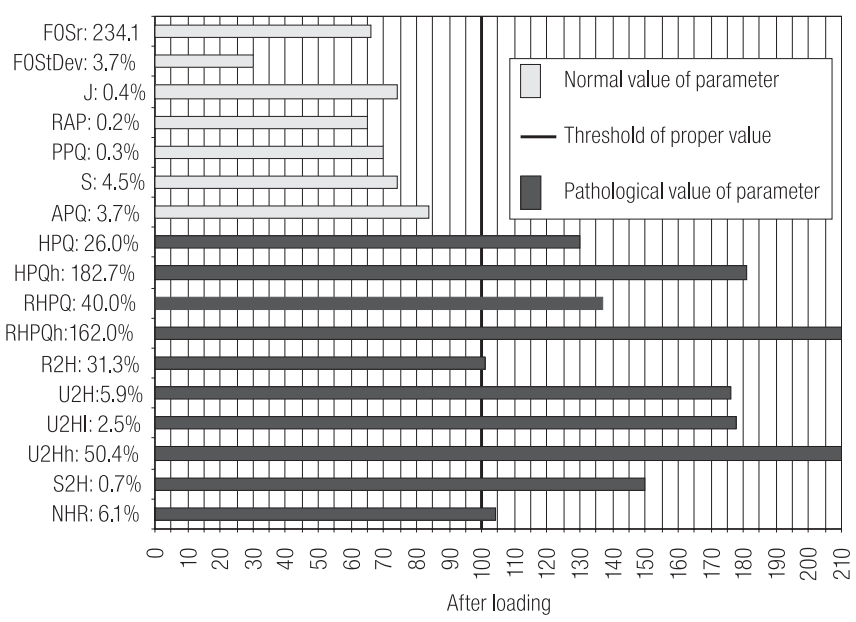

Fig. 1. The positive outcome of vocal loading in acoustic analysis - the number of pathological acoustic measurements increased by 6 after loading test. Description of parameters in text (more than 2 abnormal parameters indicate dysphonic voice). 


\section{RESULTS}

The study of VHI-score distribution showed that $66 \%$ of female teachers estimated their own voice problems as moderate disability, while $10 \%$ of the subjects reported severe voice disability (Fig. 3). In VHI outcome, 27\% of the patients were found to suffer from vocal fatigue. The assessment of maximum phonation time revealed that its average value for the total group was $13 \mathrm{~s}$ (range, 5-35 s). However, the standard maximum phonation time is $20-25 \mathrm{~s}$ and a decrease in this aerodynamic parameter below $15 \mathrm{~s}$ shows an insufficiency of voice apparatus.

The laryngovideostroboscopic evaluation enabled diagnosis of voice pathologies in the examined female teachers as functional (reversible) voice disorders. The most frequent (35.2\%) non-organic pathology observed in LVSS in the examined group was glottal insufficiency with bowed vocal folds. Soft vocal nodules (prenodules) were found in $31.4 \%$ and hyperfunctional dysphonia with tendency to vestibular phonation in $19.6 \%$ of the patients (Table 1). Discrete disturbances of the parameters estimating phonation function were observed in the initial LVSS; the most frequent abnormalities were incorrect glottal closure (hour-glass-shaped closure - characteristic of prenodules, spindle glottal closure - characteristic of glottal insuficiency with bowed vocal folds, and hyperclosure with tendency to adduct vestibular folds - characteristic of severe hyperfunctional dysphonia) that was found in $88 \%$ of the patients. Irregular vocal fold vibration was observed in $76 \%$, abnormal vibra-

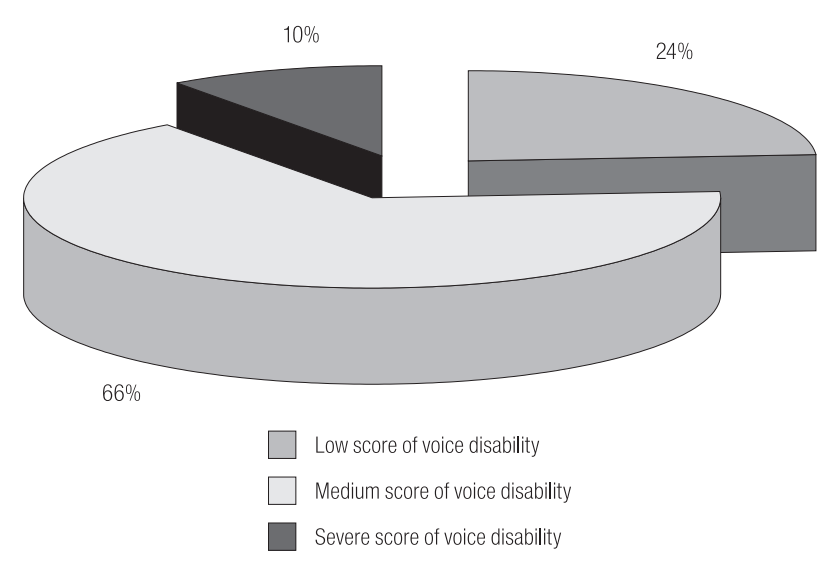

Fig. 3. The voice handicap index (VHI) score distribution in the teachers.
Table 1. Voice disorders diagnosed in the female teachers

\begin{tabular}{lcc}
\hline \multicolumn{1}{c}{ Voice disorder } & $\mathrm{n}$ & $\%$ \\
\hline Soft vocal nodules (prenodules) & 16 & 31.4 \\
Glottal insufficiency with bowed vocal folds & 18 & 35.2 \\
Hyperfunctional dysphonia with vestibular vox & 10 & 19.6 \\
Others (edema, hemorrhage) & 7 & 13.8 \\
\hline
\end{tabular}

tion amplitude in $70 \%$, and abnormal mucosal wave in $64 \%$ of the patients. The reaction of the patients to vocal loading in respect of LVSS parameters varied widely; in some patients the abnormalities were more severe, in others they remained at the same level, while some other subjects showed an improvement (i.e., incompletness of glottal closure increased in $18 \%$, decreased in $18 \%$ and remained at the same level in $64 \%$ of the patients; mucosal wave did not change in 58\%, improved in $26 \%$ and deteriorated in $16 \%$ of the patients). Nevertheless, the visible changes in the videostroboscopic parameters after the vocal-loading test did not reach the level of statistical significance.

The results of acoustic analysis performed before and after the vocal-loading test differed significantly, particularly with respect to the frequency of perturbation parameters. Jitter, RAP and PPQ increased significantly in the whole study group (Table 2). The same was true of P-I contour of vowel /a:/ that increased significantly $(p=0.04)$ in all patients after vocal loading. This finding indicated voice instability during sustained phonation in examined female teachers. However, the amplitude perturbation parameters (Shimmer and APQ) after vocal loading did not change significantly in the whole group. The Shimmer increased significantly exclusively in the hyperfunctional dysphonia subgroup (Table 3). Shimmer results after vocal loading test varied between the subgroups depending on diagnosis and this difference also reached the level of statistical significance at $\mathrm{p}_{1}=0.045$ (Table 3).

The comparison of individual acoustic analysis data performed before and after vocal loading showed that not all of the teachers experienced deterioration in individual acoustic parameters; in some they remained unchanged, while in few others they even improved. However, for Jit- 
Table 2. Frequency perturbation parameters before and after the vocal-loading test

\begin{tabular}{lcccc}
\hline Parameter & Before loading & After loading & Normal value & P \\
\hline Jitter & 0.27 & 0.31 & up to 0.5 & $0.02^{*}$ \\
RAP & 0.17 & 0.18 & up to 0.3 & $0.04^{*}$ \\
PPQ & 0.19 & 0.22 & up to 0.3 & $0.01^{*}$ \\
\hline
\end{tabular}

$* \mathrm{P}<0.05 ;$ Jitter - pitch perturbation quotient;

$\mathrm{RAP}$ - relative average perturbation; $\mathrm{PPQ}$ - pitch period perturbation quotient.

Table 3. Shimmer before and after the vocal-loading test with respect to diagnosis

\begin{tabular}{|c|c|c|c|c|c|}
\hline Diagnosis & $\begin{array}{l}\text { Before } \\
\text { loading }\end{array}$ & $\begin{array}{c}\text { After } \\
\text { loading }\end{array}$ & $\begin{array}{c}\text { Normal } \\
\text { value }\end{array}$ & $\mathrm{P}$ & $\mathrm{P}_{1}$ \\
\hline Vocal nodules & 4.1 & 4.1 & & 0.91 & \\
\hline Glottal insufficiency & 4.4 & 4.0 & 4.6 & 0.12 & $0.045^{*}$ \\
\hline $\begin{array}{l}\text { Hyperfunctional } \\
\text { dysphonia with a } \\
\text { tendency towards } \\
\text { vestibular phonation }\end{array}$ & 3.9 & 4.7 & & $0.05^{*}$ & \\
\hline Total & 4.15 & 4.2 & & 0.53 & \\
\hline
\end{tabular}

*P $<0.05 ; \mathrm{P}_{1}<0.05$ (Shimmer results after the vocal-loading test also varied between the subgroups).

Table 4. Number of subjects with significant changes in the value of acoustic characteristics after vocal loading

\begin{tabular}{lcccc}
\hline \multicolumn{1}{c}{ Parameters } & $\begin{array}{c}\text { Improvement } \\
\text { (No. of } \\
\text { patients) }\end{array}$ & $\begin{array}{c}\text { Deterioration } \\
\text { (No. of } \\
\text { patients) }\end{array}$ & $\begin{array}{c}\text { No change } \\
\text { (No. of } \\
\text { patients) }\end{array}$ & P \\
\cline { 1 - 3 } $\begin{array}{l}\text { Pitch-Intensity } \\
\text { contour of vowel /a:/ }\end{array}$ & 18 & 32 & 1 & $0.05^{*}$ \\
Jitter & 11 & 19 & 21 & $0.04^{*}$ \\
PPQ & 7 & 15 & 26 & $0.03^{*}$ \\
\hline
\end{tabular}

${ }^{*} \mathrm{P}<0.05$; Jitter - pitch perturbation quotient; $\mathrm{PPQ}$ - pitch period perturbation quotient.

ter, PPQ and P-I contour of vowel /a:/, changes (deterioration) were found in a significant number of teachers (Table 4).

\section{DISCUSSION AND CONCLUSIONS}

The effect of vocal loading on acoustic parameters was studied in professionally active, full-time female teachers with functional dysphonia. Functional (non-organic) voice disorders are often found in persons with vocally demand- ing professions and are characterized by vocal fatigue and reduced vocal endurance. The vocal abuse syndromes in professional voice users were described by Koufman [16] as common primary muscle tension patterns (MTPs). This condition may contribute to hyperfunction of vocal organ and phonation with imbalanced or excessive muscular forces. The most common laryngeal manifestation of vocal hyperfunction includes vocal nodules, bowed vocal folds with glottal insufficiency, and excessive vocal fold tension with vestibular folds approximation. In our group of female teachers with voice disorders, bowing vocal fold with incomplete glottal closure was observed in $35.2 \%$, soft vocal nodules (prenodules) in $31.4 \%$ and hyperfunctional dysphonia with a tendency towards vestibular phonation in $19.6 \%$ of the patients.

Symptoms of functional dysphonia often occur during or after vocal loading. Therefore, in our study we subjected the dysphonic teachers to the vocal-loading test to provoke vocal fatigue. After vocal loading, several acoustic parameters in our patients showed statistically significant deterioration $(\mathrm{p}<0.5)$ compared to pre-test outcomes. The most pronounced differences were found in the frequency perturbation parameters: Jitter $(p=0.02)$ RAP $(p=0.04)$, and PPQ $(p=0.01)$. However, the values of these parameters did not exceed the upper threshold of the standard, but their statistically significant deterioration may point to deviations in the voice microstructure. These findings are in agreement with previous studies performed among patients with functional voice disorders [11]. After vocal loading we also observed statistically significant deterioration (increase) of P-I contour of vowel /a:/, an indication of voice instability. The increased area of P-I contour for vowel /a:/ after vocal effort might represent a sign of vocal fatigue along with associated compensation disturbances. Kotby et al. [17] reported fundamental frequency instability in functional dysphonia. They argued that this symptom was related to the severity of dysphonia. Jilek et al. [18] investigating vocal instability in functional dysphonia, found frequency perturbations more pronounced in hypertonic dysphonia than in hypotonic and euphonic (normal) voices. In our group, the teachers with hyperfunctional dysphonia and a tendency towards vestibular phonation 
showed more acoustic disturbances after vocal loading, not only frequency perturbations like those noted in the whole study group, but also amplitude perturbations. The increase in Shimmer after the vocal-loading test for this subgroup was statistically significant, $\mathrm{p}=0.05$. Jilek et al. [18] also reported that vocal loading induced different reactions in dysphonic voices, some voices showed increased perturbations, while others showed no perturbation or even some improvement. In some patients of our group, a reaction of functional improvement after vocal loading, similar to that described above, was observed. However, some acoustic parameters (Jitter, PPQ and P-I contour of sustained vowel /a:/) deteriorated after the vocal-loading test in a significant number of the teachers $(p<0.05)$. Investigations should accommodate possible individual differences in the response to occupational and other voice demands.

To sum up, after vocal loading, some voice acoustic parameters in the teachers with functional dysphonia displayed statistically significant abnormalities, mostly in frequency perturbation parameters (Jitter's group) and P-I contour of vowel /a:/, an indication of voice instability. Acoustic analysis performed before and after the vocal-loading test can significantly contribute to objective voice examinations and may also be useful in diagnosis of early stages of dysphonia in teachers or other professional voice users. Further investigations of factors that promote individual susceptibility to vocal fatigue are necessary.

\section{REFERENCES}

1. Titze IR, Lemke J, Montequin D. Populations in the U.S. workforce who rely on voice as a primary tool of trade: A preliminary report. J Voice 1997;11(3):254-9.

2. Roy N, Merrill RM, Thibeault S, Parsa R, Gray SD, Smith EM. Prevalence of voice disorders in teachers and general population. J Speech Lang Hear Res 2004;47:281-93.

3. Śliwińska-Kowalska M, Niebudek-Bogusz E, Fiszer M, Łoś-Spychalska T, Kotyło P, Sznurowska-Przygocka B. The prevalence and risk factors for occupational voice disorders in teachers. Folia Phoniatr Logop 2006;58(2):85-102.
4. Obrębowski A, Pruszewicz A, Sułkowski W, Wojnowski W, Sinkiewicz A. Proposal of rational procedures for certifying occupational voice diseases. Med Pr 2001;52:35-44 [in Polish].

5. Vilkman E. Occupational safety and health aspects of voice and speech professions. Folia Phoniatr Logop 2004;56 (4):220-53.

6. Rubin JS, Sataloff RT, Korovin GS. Diagnosis and Treatment of Voice Disorders. New York: Thomson Delmar Learning; 2003.

7. Holmberg EB, Hillman RE, Perkell JS, Guiod P, Goldman SL. Comparisons among aerodynamic, electroglottographic, and acoustic spectral measures of female voice. J Speech Hear Res 1995;38: 1213-23.

8. Yu P, Ouaknine M, Revis J, Giovanni A. Objective voice analysis for dysphonic patients: A multiparametric protocol including acoustic and aerodynamic measurements. J Voice 2001;15(4):529-42.

9. Speyer R, Wieneke GH, Dejonckere PH. Documentation of progress in voice therapy: perceptual, acoustic, and laryngostroboscopic findings pretherapy and posttherapy. J Voice 2004;18 (3):325-40.

10. Wiskirska-Woznica B. The complex voice evaluation in organic and functional larynx disorders. [PhD Thesis]. Poznań: University of Medical Science; 2002 [in Polish].

11. Swidzinski P. Usefulness of acoustic analysis in diagnostics of voice disorders [PhD thesis] Poznań: University of Medical Science; 1998 [in Polish].

12. Awan SN, Roy N. Acoustic prediction of voice type in women with functional dysphonia. J Voice 2005;19(2):268-82.

13. Chang A, Karnell MP. Perceived phonatory effort and phonation threshold pressure across a prolonged voice loading task: A study of vocal fatigue. J Voice 2004;18(4):454-66.

14. Simberg S, Sala E, Tuomainen J, Sellman J, Rönnemaa AM. The effectiveness of group therapy for students with mild voice disorders: a controlled clinical trial. J Voice 2006;20(1):97-109.

15. Roy N, Hendarto H. Revisiting the pitch controversy: changes in speaking fundamental frequency (SFF) after management of functional dysphonia. J Voice 2005;19(4):582-91.

16. Koufman JA. Laryngeal muscle tension patterns (MTPs) In: Diagnosis and Treatment of Voice Disorders. Rubin JS, Sataloff RT, Korovin GS, editors. New York: Thomson Delmar Learning; 2003. pp. 175-82.

17. Kotby MN, Titze IR, Saleh MM, Berry DA. Fundamental frequency stability in functional dysphonia. Acta Otolaryngol 1993;113:439-44.

18. Jilek C, Marienhagen J, Hacki T. Vocal stability in functional dysphonic versus healthy voices at different times of voice loading. J Voice 2004;18(4):443-53 